\title{
Neurosurgery during the COVID-19 pandemic: update from Lombardy, northern Italy
}

\author{
Cesare Zoia ${ }^{1}$. Daniele Bongetta ${ }^{2} \cdot$ Pierlorenzo Veiceschi $^{3} \cdot$ Marco Cenzato $^{4} \cdot$ Francesco Di Meco $^{5} \cdot$ Davide Locatelli $^{6}$. \\ Davide Boeris ${ }^{4}$. Marco M. Fontanella ${ }^{7}$
}

Received: 21 March 2020 / Accepted: 23 March 2020 / Published online: 28 March 2020

(C) Springer-Verlag GmbH Austria, part of Springer Nature 2020

Since February, 21st 2020, when the first person infected was reported in Lombardy, Italy rapidly became home to a massive Coronavirus Disease 2019 (COVID-19) outbreak. Currently, on 21th March, 53,578 COVID-19 cases have been confirmed in Italy. 6072 patients are now hospitalized. The number of deaths has risen to 4825 while 6072 were declared healed [4]. This data shows that Italy is, currently, the second most affected nation in the world by the epidemic, second only to China.

Most of these cases occurred in Lombardy, the most populated Region of Italy, accounting for 10,060,574 people [2] . Hospitals were rapidly overcrowded by COVID-19 patients, especially intensive care units, and non-specialized doctors in infectious or respiratory diseases, including Neurosurgeons, were reassigned to the new COVID-wards to rationalize the use of resources. Hence, the regional health system, has been rapidly reprogrammed trying to contain the COVID-19 $[1,3]$. In particular, for this reason, on March, 8th 2020, the Lombard

Pierlorenzo Veiceschi

pierloveiceschi@gmail.com

1 Department of Neurosurgery, Fondazione IRCCS Policlinico San Matteo, Pavia, Italy

2 Department of Neurosurgery, ASST Fatebenefratelli Sacco, Milan, Italy

3 Department of Neuroscience and Surgery of the Nervous System, ASST Papa Giovanni XXIII Hospital, Piazza OMS, 1, 24127 Bergamo, Italy

4 Department of Neurosurgery, Grande Ospedale Metropolitano Niguarda, Milan, Italy

5 Department of Neurosurgery, Carlo Besta Institute of Neurology, IRCCS and Foundation, Milan, Italy

6 Division of Neurosurgery, Ospedale di Circolo ASST Sette Laghi, University of Insubria, Varese, Italy

7 Division of Neurosurgery, Department of Surgical Specialties, Radiological Sciences and Public Health, University of Brescia, Brescia, Italy
Regional Council organized an emergency task force in order to lead the response to the outbreak.

This viewpoint is intended to summarize the reorganization model provided inside the Lombard Neurosurgical network. Neurosurgeons and other non-specialized doctors were reassigned to the new COVID-wards. The Health system worked trying to contain COVID-19 in the region. The Lombard Regional Council called an emergency task force in order to reprogram the regional system. The Lombardy region accounts for 26 neurosurgical departments in 21 hospitals with more than 200 neurosurgeons and 40 residents in training. During the early days of the first outbreak, each neurosurgical department responded individually, and according to the number of COVID-19 positive patients present in the hospital, elective surgical activities, hospitalizations, and nonurgent outpatient visits were gradually reduced. Since March 8th 2020, the regional medical system, including neurosurgical activities, has been completely reorganized by the decision of the Lombard Regional Government (decree ${ }^{\circ} \mathrm{XI} /$ 2906).

The regional task force has determined that all non-urgent outpatient activities had to be suspended. It was decided to remodel the hospital treatment system by identifying 4 neurosurgical "hub" hospitals where concentration of all neurosurgical activities that could not be postponed would take place.

Three hub hospitals guarantee 24/7 acceptance of emergency cases. The three hospitals have been chosen on geographical bases, covering roughly $1 / 3$ of Lombard territory divided in west, central and east, all of the other departments have been assigned to one of the three hubs as a "spoke".

The fourth "hub" hospital, the regional neuro oncological center has been re-allocated for urgent oncological patients coming from all the other departments of the region.

Each department also provided some of its own neurosurgeons to be assigned to increase the staff on duty at the relative hub, accounting for personnel availability. The following clinical situations have been defined as neurosurgical emergencies 
- cerebral hemorrhages (subarachnoid and intraparenchymal) acute hydrocephalus - tumors at risk of intracranial hypertension - spinal cord compressions with neurological deficit or at risk of - traumatic cranial and spinal trauma emergencies.

The patients may access the hub in two ways: by primary or secondary transport. Primary transport is advised when the access is direct into the hub from the territory, the patient is evaluated by the neurosurgeon on duty, undergoes the necessary diagnostic procedures and, on symptomatic patients who need emergency intervention or hospitalization in intensive care, a COVID-19 swab is routinely carried out.

Secondary transport: the patient is evaluated at the "spoke" department. If considered an urgent case, he/she is centralized to the hub hospital after the execution of a swab for COVID19.

Dealing with oncological pathology, priority criteria have been defined:

1) Class $\mathrm{A}++$ (requiring immediate treatment): patients with intracranial or spinal oncological pathology that need emergency treatment (rapidly evolving intracranial hypertension with deteriorating state of consciousness, acute hydrocephalus, spinal cord compression with rapid tetraor paraparesis).

2) Class $\mathrm{A}+$ (requiring treatment within a maximum of $7-$ 10 days): patients with oncological pathology (intracranial tumors with mass effect or with progressive neurological deficit, without deterioration of consciousness).

3) Class A (requiring treatment within a month): patients with oncological neurosurgical pathology that appears radiologically of suspected malignant nature or with oncological pathology that determines a neurological deficit.

Class A ++ patients will be managed like other emergencies, those in the other two classes will be managed by the oncological hub, according to clinical priorities and programmed in the allocated surgical slots.

"Emergency Hubs" have doubled the number of neurosurgeons on duties with the collaboration of the colleagues from the "Spoke hospitals". This was found necessary to safely manage more than one operating theatre at the time. 2 Neurosurgeons are on duty $8-20 \mathrm{~h}$ and 2 neurosurgeons are on duty $20-8 \mathrm{~h}$. There is also an on-call system to provide subspecialist cover. A complex spine on call service is provided $24 / 7$ as well as neurovascular coverage. Once the postoperative procedures have been completed, the patient will be discharged home or to a physical therapy ward if necessary.
The oncological cases proposed to the "Oncological hub" by external centers are operated by the proposing teams or by mixed teams composed of external and residential surgeons. In both cases, hub staff facilitate the execution of surgical procedures by external operators.

This organization was prepared to let the majority of the working force focus on COVID-19 patients, in the most affected areas.

Even though we cannot have definitive data in such a short period, our preliminary impressions are that this system is sustainable, at least in the short period. This has been facilitated by the public "lockdown" that has tangibly diminished the number of traumatic cases, easing the surgical burden on hub centers.

The COVID-19 emergency has forced a wider collaboration between hospitals favoring the interchange of neurosurgeons between hospitals that were once in competition, hopefully creating the basis for an interesting new standard for the Post COVID-19 period. In difficult times such as these, the cooperative spirit has risen spontaneously to previously unmet levels both inside hospitals and between different departments.

We truly hope that the COVID-19 outbreak will soon end in our beloved country and that other countries may never experience such a tragic emergency. Our thanks and thoughts are to all colleagues, and not only those in the neurosurgical community, who are risking their lives to provide the best care to COVID-19 patients.

\section{Compliance with ethical standards}

Conflict of interest The authors declare that they have no conflict of interest.

\section{References}

1. Grasselli G, Pesenti A, Cecconi M (2020) Critical Care Utilization for the COVID-19 Outbreak in Lombardy. Italy JAMA. https://doi. org/10.1001/jama.2020.4031

2. Popolazione residente al primo gennaio: Lombardia http://dati.istat. it/Index.aspx? QueryId $=18548$

3. Spina S, Marrazzo F, Migliari M, Stucchi R, Sforza A, Fumagalli R (2020) The response of Milan's Emergency Medical System to the COVID-19 outbreak in Italy. Lancet 395(10227):e49-e50

4. Track Reported Cases of COVID-19 https://coronavirus.jhu.edu/ map.html

Publisher's note Springer Nature remains neutral with regard to jurisdictional claims in published maps and institutional affiliations. 